PO079

\title{
BEAT FLICKER - A TEMPORAL LIGHT ARTEFACT DUE TO MULTIPLE SOURCES OF TIME MODULATED LIGHT \\ Johannes Lindén et al.
}

DOI 10.25039/x46.2019.PO079

from

CIE x046:2019

Proceedings

of the

29th CIE SESSION

Washington D.C., USA, June 14 - 22, 2019

(DOI 10.25039/x46.2019)

The paper has been presented at the 29th CIE Session, Washington D.C., USA, June 14-22, 2019. It has not been peer-reviewed by CIE.

(C) CIE 2019

All rights reserved. Unless otherwise specified, no part of this publication may be reproduced or utilized in any form or by any means, electronic or mechanical, including photocopying and microfilm, without permission in writing from CIE Central Bureau at the address below. Any mention of organizations or products does not imply endorsement by the CIE.

This paper is made available open access for individual use. However, in all other cases all rights are reserved unless explicit permission is sought from and given by the CIE.

CIE Central Bureau

Babenbergerstrasse 9

A-1010 Vienna

Austria

Tel.: +4317143187

e-mail: ciecb@cie.co.at

www.cie.co.at 


\title{
BEAT FLICKER - A TEMPORAL LIGHT ARTEFACT DUE TO MULTIPLE SOURCES OF TIME MODULATED LIGHT
}

\author{
Lindén, J., Dam-Hansen, C., Thorseth, A. \\ DTU Fotonik, Department of Photonics Engineering, Technical University of Denmark, Roskilde, \\ DENMARK \\ johli@fotonik.dtu.dk
}

DOI 10.25039/x46.2019.PO079

\begin{abstract}
Metrics of temporal light artefacts (TLAs) are tools meant to quantify the magnitude of the impact of temporal modulated light generated by light sources. However, as lighting systems are becoming more and more complex, just assigning a TLA metrics to a light source is not necessarily an easy task. For example, a luminaire containing two drivers operating on slightly different frequencies could generate a light intensity waveform, resulting in some TLA values at one moment, and different TLA values at another moment. In acoustics, the corresponding phenomena would be two tones with slightly different frequencies, giving rise to something called "beats". The authors of this paper therefore calls the "light"-version of this phenomena beat flicker. The objective of the present work is to draw attention to a challenge that might arise, when assessing temporal modulated light, using tools currently available. The presence of beat flicker could be such a challenge to handle.
\end{abstract}

Keywords: Beat Flicker, Flicker, Temporal Light Artefacts, Temporal Light Modulation, Stroboscopic effects, Stroboscopic Visibility Measure, Flicker Index, Modulation Depth, Multiple Sources

\section{Motivation, specific objective}

Temporal light artefacts (TLAs), which includes flicker, stroboscopic effect and phantom arrays, i.e. undesired visual perception effects caused by temporal light modulation (TLM) of a light source, has shown to be a threat to wider SSL adoption especially related to dimming functions and low-quality LED products. This is due to both the noticeable and unperceivable effects of TLM on human perception and wellbeing (Wilkins, 2010; Kelly, 1964; Perz, 2013; Bullough, 2011; Perz, 2014, Roberts 2012). Currently, no standards for assessing TLAs exists, however several metrics have been suggested the latest years, e.g. the stroboscopic visibility measure (SVM) in 2016 (CIE 2016). However, all the suggested metrics assumes a temporal modulated light intensity with fairly high frequencies. For example, modulation depth (MD) and flicker index ( $\mathrm{FI}$ ) originates from a time when modulation of light intensity occurred at $50 \mathrm{~Hz}$ (or $60 \mathrm{~Hz}$ ), and SVM deals with frequencies from about 100 to $2000 \mathrm{~Hz}$. These measures do not take into account the effect that could arise from several light sources with similar, but not identical, waveforms and/or frequencies. This could result in a superposition waveform, with periodic behaviour with a frequency of only a few $\mathrm{Hz}$, giving rise to a phenomenon, which in this work is called beat flicker.

Since the temporal characteristics of any LED based lighting product are directly mimicked by the characteristics of the electronic driver, any light source comprising more than one driver could, in principle, generate a superposition waveform that turns out not to be so straight forward to asses. Depending on the number of temporal modulated light sources involved, and depending on the difference in frequencies from the same, the measured values of the different TLA metrics - let it be stroboscopic visibility measure, modulation depth or whatever - will vary with time. For example, the recommended sampling duration for assessing stroboscopic visibility with SVM is 1 second. If two frequencies differ with $1 \mathrm{~Hz}$, the resulting waveform will exhibit different shapes from one second to another.

In the present work, waveforms as such, both authentic and simulated, are investigated with respect to several TLA metrics. The objective is to draw attention to the challenge of presence 
of beat flicker, which might arise in the case of a single luminaire or for that matter in the case of an entire lighting environment containing several light sources.

\section{Methods}

In the present work, both authentic and simulated superposition waveforms are investigated with respect to several TLA metrics. In the authentic case, two ceiling luminaires with slightly different frequencies of pulse width modulation are investigated. In the simulated case, two subcases of waveforms are investigated - sinusoidal and square - in order to estimate and analyse some worst-case scenarios.

The waveforms (with a duration of 20 seconds) from both the authentic and simulated scenario are analysed with respect to stroboscopic visibility measure (SVM), flicker index (FI) and modulation depth (MD), at different starting positions in time. The duration of the part of the waveform being analysed - the measurement window - is (when other is not stated) 1 second, as recommended when assessing SVM.

\subsection{Case 1: Authentic luminaires}

Figure 1 shows the ceiling luminaires of the authentic case. They are $60 \times 60 \mathrm{~cm}$ LED panels, dimmable with pulse width modulation. The frequencies of the two luminaires differs slightly: $551.8 \mathrm{~Hz}$ and $546.7 \mathrm{~Hz}$. During the measurement, the dimming levels are set close to $50 \%$, meaning both square waveforms exhibit a duty cycle close to $50 \%$. Light intensity variations from each of the lamps and both together are recorded for 20 seconds, using a photodiode and an amplifier connected to a data acquisition unit and a computer. The sample rate is $100.000 \mathrm{~S} / \mathrm{s}$.

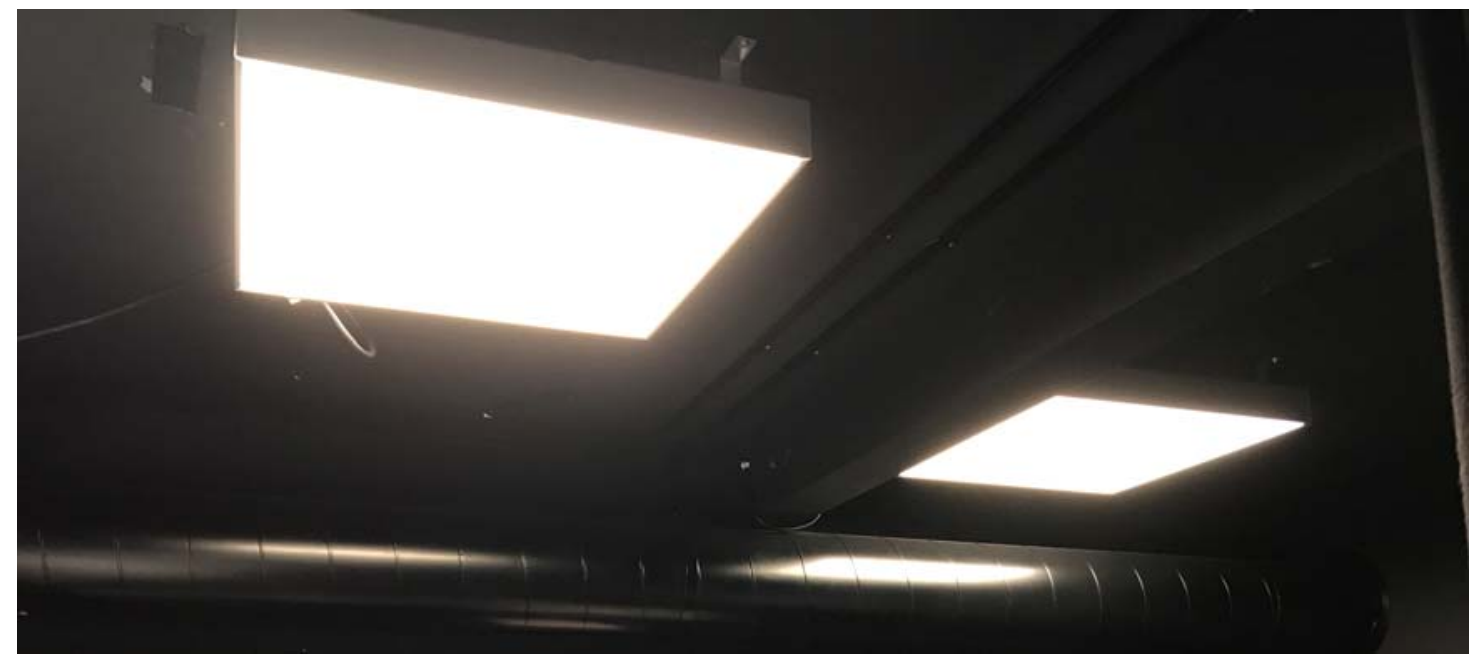

Figure 1 - Two ceiling lamps in a facility at DTU used as an authentic example. The lamps are dimmable using pulse width modulation, however using slightly different frequencies. The left lamp exhibit $551.8 \mathrm{~Hz}$ and the right lamp exhibit $546.7 \mathrm{~Hz}$.

Figure 2 shows the waveforms from the two individual lamps and two examples of how the superposition of the two individual waveforms looks at different positions in time. 

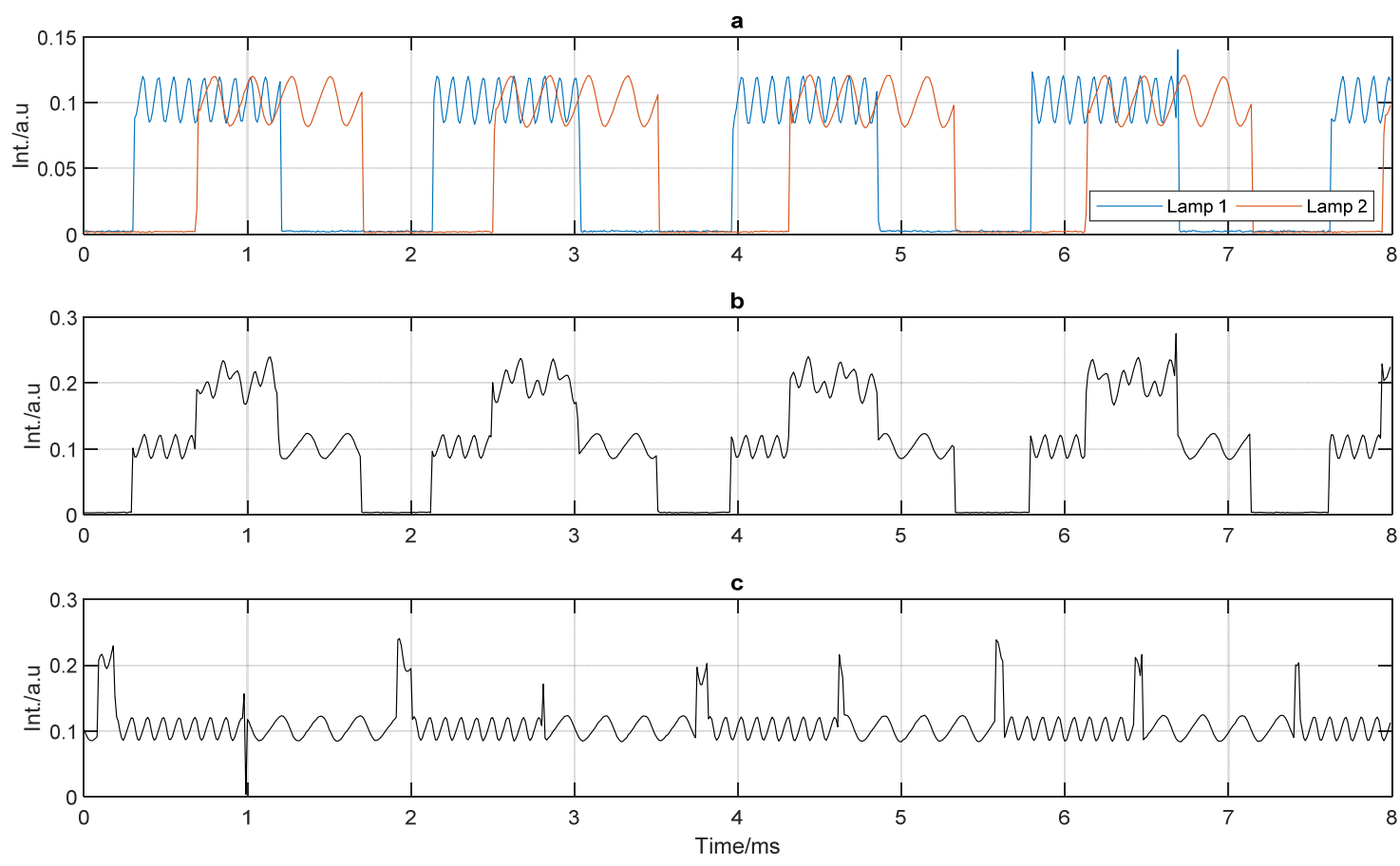

Figure 2 - a) Separated light intensity waveforms form lamp $1 @ 546.7 \mathrm{~Hz}$ and lamp 2 @ $551.8 \mathrm{~Hz}$. b) Superposition of the two waveforms in a. c) Superposition of waveforms about $130 \mathrm{~ms}$ later compared to $\mathrm{b}$.

\subsection{Case 2: Simulated waveforms}

As mentioned, two subcases of simulated waveforms are investigated: sinusoidal and square shaped waveforms. In each subcases, two waveforms are combined, one with frequency $f$ and the other with frequency, $f+\Delta$ freq. Some different cases of $f$ and $\Delta$ freq are investigated. For the sinusoidal subcase, the modulation depth is set to $50 \%$. For the square waveform subcase, the modulation depth is set to $100 \%$ and different duty cycle of 20,50 and $80 \%$ are investigated.

All simulated waveforms have a duration of 20 seconds.

\section{Results}

Below follows first the results from the measured, authentic waveforms. Then follows the result of the simulated waveforms.

\subsection{Results of measured waveforms}

Since the frequency difference from the two ceiling luminaires is about $5 \mathrm{~Hz}$, it is expected that the superposition waveform of the two exhibit a periodic behaviour with the frequency of $5 \mathrm{~Hz}$. This means that if the waveform is assessed with respect to SVM, for which the recommended measurement window is 1 second, the resulting SVM values will vary very little depending on starting point. With a measurement window of 1 second, the SVM values becomes more or less constant, with a value of $0.93 \pm 0.01$. The $\mathrm{FI}$ also become very constant, with a value of $0.27 \pm 0.003$. The MD is constant at $100 \%$ and shows no variation at all, which is expected with respect to how $M D$ is defined, and that during 1 second the waveform always reached the level of no light output.

However, if the measurement window is decreased to 0.1 seconds, strong oscillations in the resulting time resolved SVM and FI values are observed, see Figure 3. 

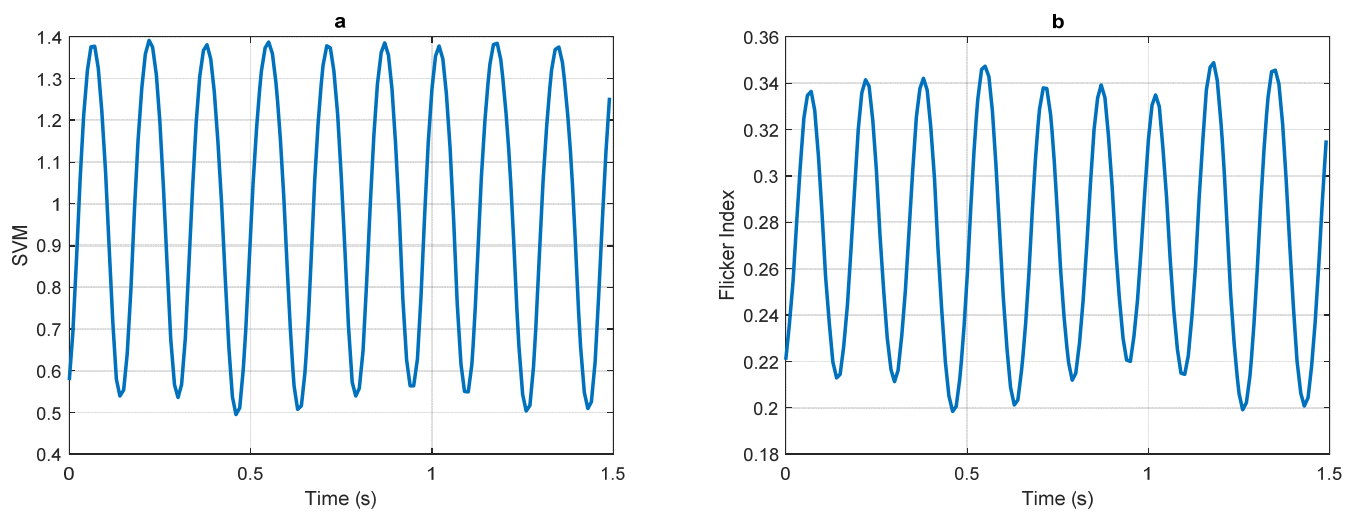

Figure 3 - Time resolved SVM (a) and FI (b) using a measurement windows size of 0.1 seconds.

Worth noticing is that the SVM and FI (using a time window of 1 second) for the waveforms from the individual lamps are 1.70 and 0.53 for lamp 1 and 1.18 and 0.37 for lamp 2, respectively (the difference between the lamps is due to the slightly different settings in dimming level). This is far higher than the SVM and FI from the waveforms combined ( 0.93 and 0.27 , respectively). I.e. it is possible to combine waveforms with slightly different frequencies to delibirately achieve lower TLA values. Weather this is a good stategy or not is too early to tell, since it cannot be excluded that the resulting temporal modulated light could have a non-visual effect on humans.

\subsection{Results of simulated waveforms}

The following section presents the results from the assessment of the simulated waveforms, first the sinusoidal ones, then the square waveforms.

\subsubsection{Sinusoidal waveforms}

Regarding the simulated sinusoidal waveforms, a frequency of $500 \mathrm{~Hz}$ and a modulation depth of $50 \%$ is selected as a starting point. Four different cases of $\Delta$ freq is investigated: $0 \mathrm{~Hz}$ (i.e. equivalent to one single sinusoidal waveform), $0.2 \mathrm{~Hz}, 1 \mathrm{~Hz}$ and $10 \mathrm{~Hz}$.

For example, in the case of $\Delta$ freq $=0.2 \mathrm{~Hz}$, the two combined waveforms becomes $500 \mathrm{~Hz}$ and $500.2 \mathrm{~Hz}$. Figure 4 shows the resulting superposition waveform of these two combined waveforms, at two different positions in time, separated by $1.9 \mathrm{~ms}$.

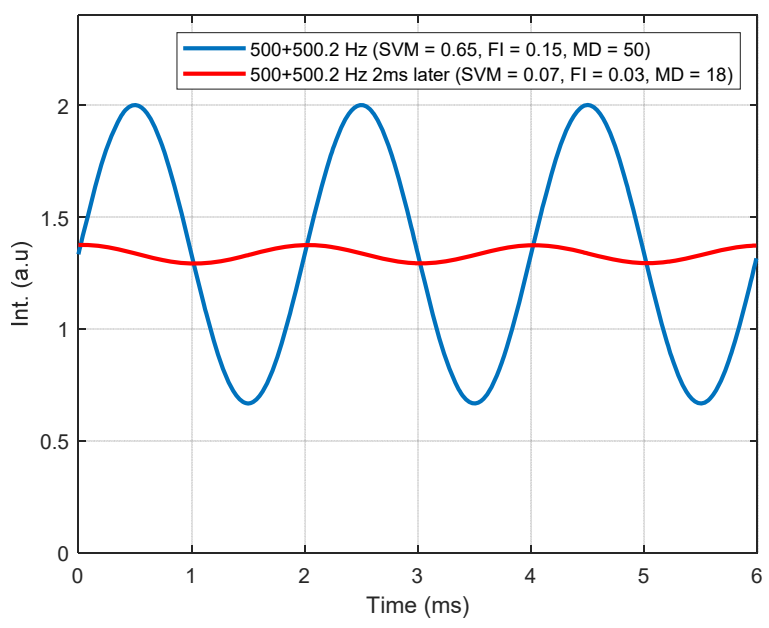

Figure 4 - Superposition of two sinusoidal waveforms of $500 \mathrm{~Hz}$ and $500.2 \mathrm{~Hz}$, at two different positions in time, generating different TLA values (shown in the legend).

As one could expect, the two waveform shapes in Figure 4 results in very different values of SVM, FI and MD (shown in the legend in the figure). Figure 5 shows the time resolved SVM, 
$\mathrm{FI}$ and MD. They are shown for combined waveforms with four different frequency differences $(0 \mathrm{~Hz}, 0.2 \mathrm{~Hz}, 1 \mathrm{~Hz}$ and $10 \mathrm{~Hz}$ ) with one of the waveforms frequency being $500 \mathrm{~Hz}$.
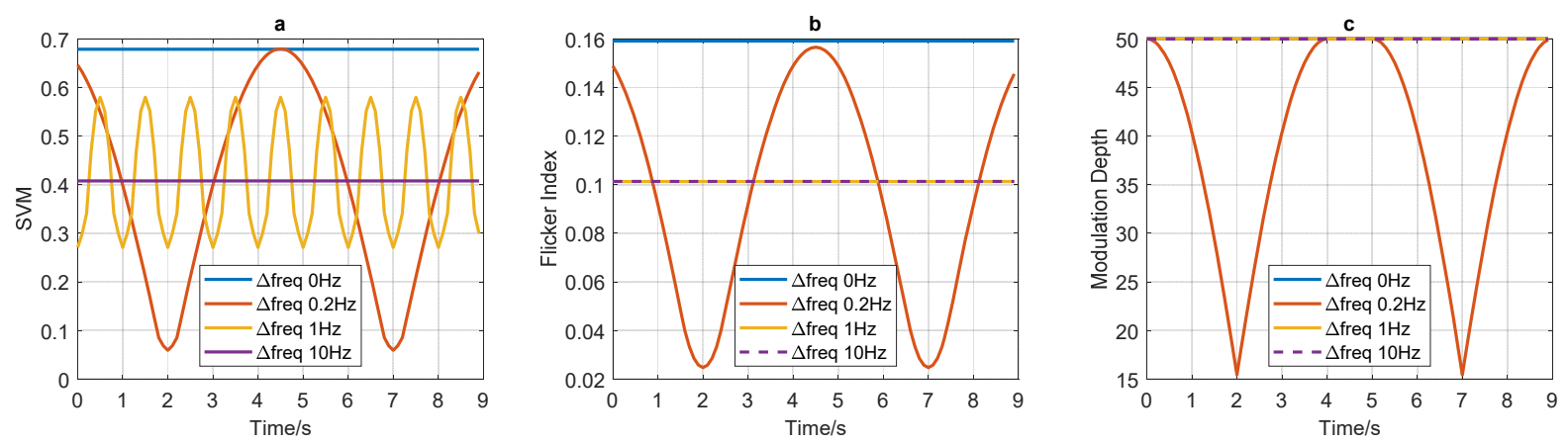

Figure 5 - Time resolved SVM (a), FI (b) and MD (c) for two superposition sinusoidal waveforms at $500 \mathrm{~Hz}$ and $500+\Delta$ freq $\mathrm{Hz}$ for four different $\Delta$ freq.

It can be seen in Figure $5 a$, that with no frequency difference the SVM is constant with a value of 0.68 (blue curve). However, as the frequency difference increases to $0.2 \mathrm{~Hz}$, the SVM value start to oscillate to as low values as 0.05 (red curve). At a frequency difference of $1 \mathrm{~Hz}$, the oscillations are decreased in amplitude (orange curve), only to stabilize around 0.41 as the frequency difference has reached $10 \mathrm{~Hz}$ (purple curve). In Figure 5b, showing the time resolved $\mathrm{FI}$, a similar behaviour can be observed. Without any frequency difference, the $\mathrm{FI}$ is constant with a value of 0.16 (blue curve). With a frequency difference of $0.2 \mathrm{~Hz}$, the FI value oscillates between 0.02 and 0.16 (red curve). For frequency differences greater than $1 \mathrm{~Hz}$, the FI has stabilized around a value of 0.10 (orange and purple curve). In Figure $5 \mathrm{c}$ it can be seen that it's only for frequency differences around $0.2 \mathrm{~Hz}$ the MD oscillates to as low values as $15 \%$ (red curve), while for other frequency differences is stays constant at $50 \%$.

As mentioned, the selected frequency of $500 \mathrm{~Hz}$ and modulation depth of $50 \%$ was only selected as a starting point. As different frequencies and modulation depths are tested, generally it is observed that a lower frequency and/or a greater modulation depth will increase the amplitude and maximum values of all TLA metrics oscillations.

Regarding different sizes of the measurement window, this is rather irrelevant for the SVM, since the recommendation for assessing SVM is 1 second. However, for FI and MD no such recommendations exists. It is observed that a shorter measurement window would result in even lower minimum values of $\mathrm{FI}$ and MD than for a measurement window size of 1 second, in those cases where oscillations occur.

\subsubsection{Square waveforms}

Also for the square shaped waveforms, a similar approach as for the sinusoidal waveforms is used: a frequency of $500 \mathrm{~Hz}$ is used as a starting point. Also as a starting point, the duty cycle of $50 \%$ is selected. The modulation depth, however, is chosen to be $100 \%$ in all cases using square waveforms, since this is the most common way to apply square waveforms. As in the case of the sinusoidal waveforms, four different cases of $\Delta$ freq is investigated: $0 \mathrm{~Hz}$ (i.e. equivalent to one single square shaped waveform), $0.2 \mathrm{~Hz}, 1 \mathrm{~Hz}$ and $10 \mathrm{~Hz}$. The influence of some different cases of duty cycles are also investigated: $20 \%, 50 \%$ and $80 \%$.

Figure 6 shows an example of a resulting superposition waveform of two combined square waveforms (here $500 \mathrm{~Hz}$ and $500.2 \mathrm{~Hz}$ ), at two positions in time separated by $2 \mathrm{~ms}$. 


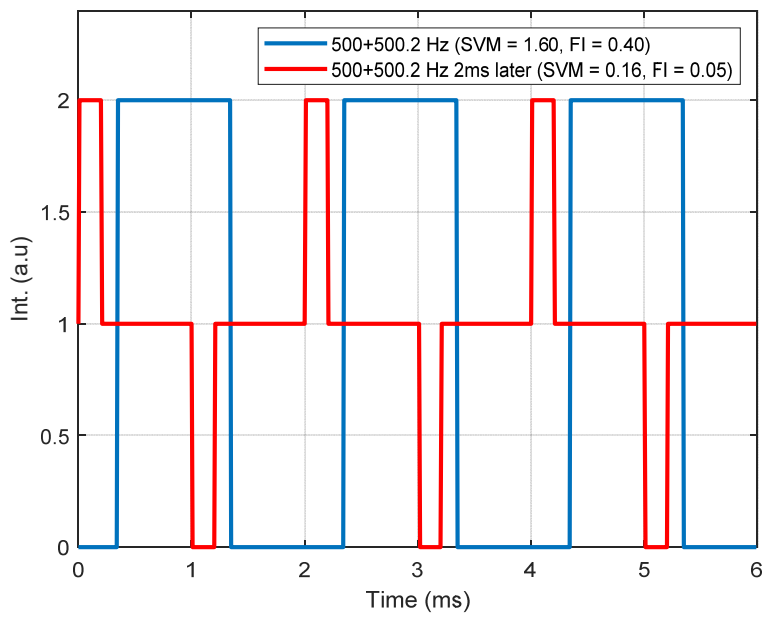

Figure 6 - Superposition of two square shaped waveforms of $500 \mathrm{~Hz}$ and $500.2 \mathrm{~Hz}$, at two different positions in time, generating different TLA values (shown in the legend).

As expected, the SVM and FI values are different depending on starting position of the measurement window. The MD, however, shows no variation in this case, and is constant at $100 \%$.

The time resolved SVM and $\mathrm{FI}$ are shown in Figure 7 for combined waveforms with four different frequency differences $(0 \mathrm{~Hz}, 0.2 \mathrm{~Hz}, 1 \mathrm{~Hz}$ and $10 \mathrm{~Hz})$.
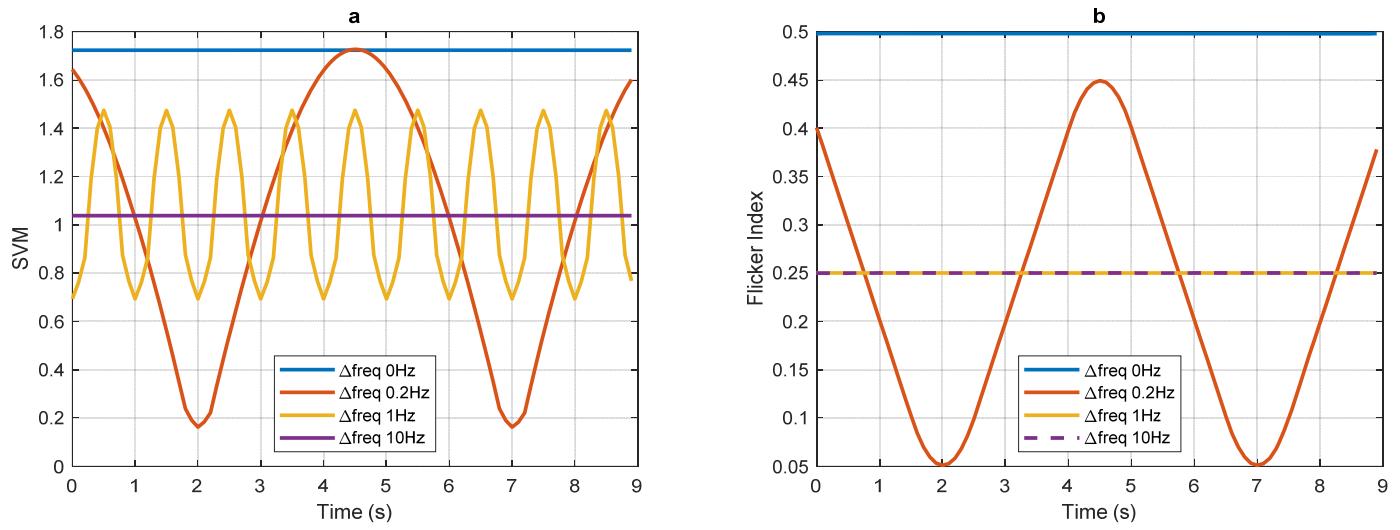

Figure 7 - Time resolved SVM (a) and FI (b) for two superposition square shaped waveforms at $500 \mathrm{~Hz}$ and $500+\Delta$ freq $\mathrm{Hz}$ for four different $\Delta$ freq.

In Figure 7a, showing the time resolved SVM values, it can be seen that the SVM is constant at both low and high frequency differences $(0 \mathrm{~Hz}$ and $10 \mathrm{~Hz}$ respectively), but at different values (1.72 and 1.04, respectively). Hence, it is possible to achieve a lower SVM value if one combine two waveforms with a frequency difference greater than $10 \mathrm{~Hz}$. Actually, a thorough investigation shows that the SVM value stabilizes already at a frequency difference of $2 \mathrm{~Hz}$. If the frequency difference is between 0 and $2 \mathrm{~Hz}$, the SVM value will oscillate, and the smaller the frequency difference, the bigger the oscillations. These frequency differences are in fact independent of the main frequency. In the case of using two square waveforms with the frequencies of 500 and $500.2 \mathrm{~Hz}$ the SVM value will oscillate between 1.73 and 0.16 (indicated by the red curve in Figure 7a) with a period time of 5 seconds (this is about as big the oscillations gets). In such a case, it is therefore a high risk of measuring a lower SVM value than the highest occurring.

A similar situation can be seen for the $\mathrm{FI}$ in Figure $7 \mathrm{~b}$. At $0 \mathrm{~Hz}$ frequency difference the $\mathrm{FI}$ is stable at 0.5 . However, already at a frequency difference of $1 \mathrm{~Hz}$ the $\mathrm{FI}$ has stabilized around 
0.25 , i.e. half the value. At a frequency difference of $0.2 \mathrm{~Hz}$ the $\mathrm{FI}$ strongly oscillates between 0.45 and 0.05 .

Changing the main frequency in the simulation above have a major effect on the SVM value: the lower the frequency the higher the SVM values and bigger the oscillations. However, changing the frequency have no effect on neither FI nor MD.

The effect of how different duty cycles effects the SVM, FI and MD is investigated. The different duty cycle studied are 20,50 and $80 \%$. The frequency waveform combination chosen is 500 and $500.2 \mathrm{~Hz}$.

Figure 8 shows the time resolved SVM and FI for the three different duty cycle cases.
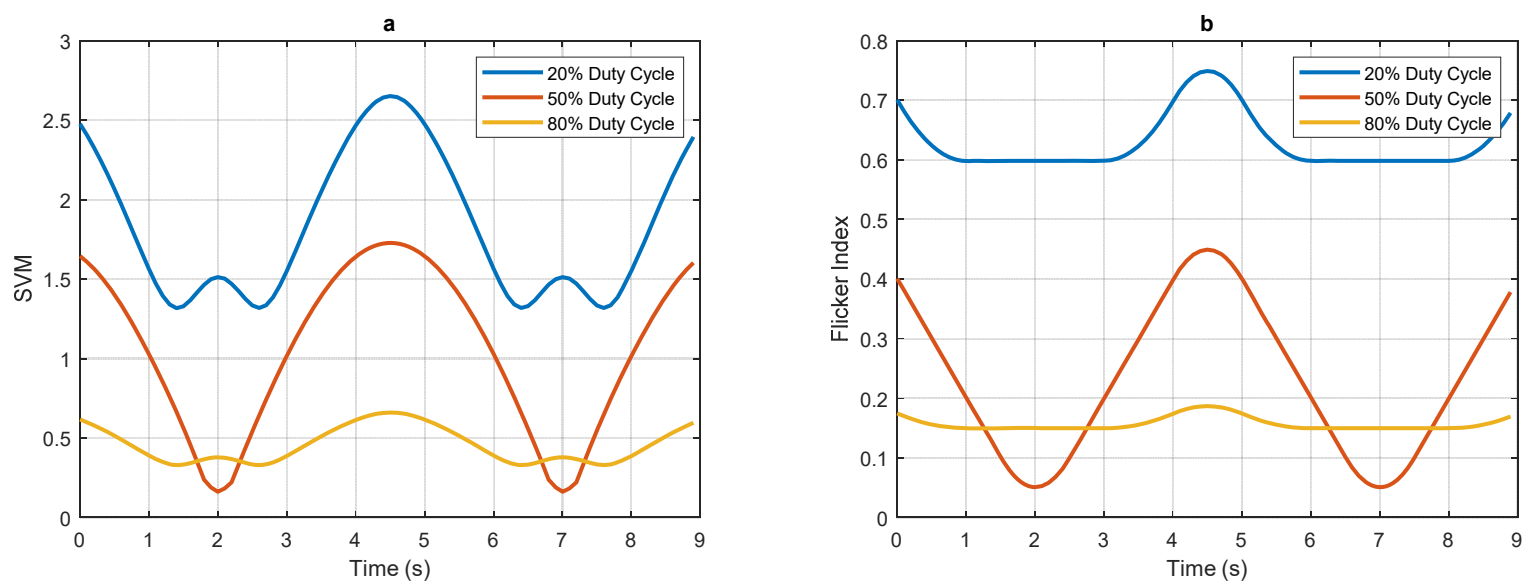

Figure 8 - Time resolved SVM (a) and FI (b) for two superposition square shaped waveforms at $500 \mathrm{~Hz}$ and $500.2 \mathrm{~Hz}$ for three different duty cycles.

In Figure $8 \mathrm{a}$ and $\mathrm{b}$ it can be seen that for a duty cycle of $20 \%$, both higher SVM and FI values are occasionally reached $(2.7$ for SVM and 0.75 for $\mathrm{FI}$ ). However, the oscillations are not as pronounced as in the case of a duty cycle of $50 \%$. On the other hand, here the absolute values are a bit lower. At a duty cycle of $80 \%$ though, both the SVM and FI starts to stabilize around lower values. The MD (not shown in the figure) is constant at $100 \%$ for duty cycles below about 50 to $60 \%$. Above that, the MD occasionally drops to $33 \%$. The duty cycle of which the MD drops to $33 \%$ depends on the size of the measurement window.

\section{Conclusions}

It is shown that light originating from a multiple light source luminaire could in some cases give rise to temporal variations in TLA metric values, due to the phenomenon in this paper called beat flicker.

In this work an authentic example, involving two luminaries using PWM at 546 and $551 \mathrm{~Hz}$ respectively, was investigated. This example unfortunately turns out being a bad example for illustrating the problem with beat flicker regarding SVM. The reason is the frequency difference between the two luminaires being to big $(5 \mathrm{~Hz})$. This in combination with the recommended measurement window of 1 second when assessing SVM does not results in any oscillations or beat flicker. However, assessing FI or MD, no such recommendations on measurement window size exists. Using a measurement window of 0.1 seconds shows that the resulting FI value will vary between 0.2 and 0.35 , depending on starting position.

Regarding the investigation of the simulated sinusoidal waveforms, two important conclusions can be drawn from Figure 5. First: By introducing a small frequency difference between two waveforms, all the TLA metrics SVM, FI and MD occasionally will drop to significantly lower values than would for a single waveform with the same frequency. Second, with a frequency difference greater than $5 \mathrm{~Hz}$ between two waveforms, the resulting SVM and $\mathrm{FI}$ value will stabilize at a significantly lower value, than would for a single waveform with the same frequency. 
It is observed that a shorter measurement window would result in even lower minimum values of $\mathrm{FI}$ and MD than for a measurement window size of 1 second, in those cases where oscillations do occur. This is rather irrelevant for the SVM, since the recommendation for assessing SVM is 1 second. However, for $\mathrm{FI}$ and MD no such recommendations exists. This concludes that if a too short part of light intensity waveform are to be investigated, and this light originates from a multiple light source luminaire, there is a risk of too low readings of $\mathrm{FI}$ and MD values.

Regarding the investigation of the simulated square shaped waveform, it is concluded that combining two waveforms with a frequency difference greater than $2 \mathrm{~Hz}$, it is possible to achieve a lower SVM value than using only one similar waveform at the same frequency.

Investigating the influence of different duty cycles, the conclusion is that the biggest oscillations in the SVM and FI are observed at duty cycle of about $50 \%$, provided the frequency difference between the two waveforms are smaller than $2 \mathrm{~Hz}$.

For both the measured and the simulated cases, it is shown that combining two waveforms, each of which results in high TLA, with each other using slightly different frequencies, the TLAs becomes less articulated. However, as mentioned, it is too early to tell weather this is a recommended stategy to practice in order to achieve lower TLA values, since it cannot be excluded that the resulting temporal modulated light still could have a non-visual effect.

One important conclusion of this work is that in order to fairly assess TML, and to get a justified result of the TLAs, it is necessary to make a more thorough investigation of the waveform in question. If the waveform exhibit periodic changes with frequencies below $1 \mathrm{~Hz}$, values of e.g. SVM might vary significantly depending on which part of the waveform being assessed. These types of artefacts (variation of TLA values in time) could be difficult to detect using hand held TLA measurement devices. The possibility of beat flicker should be taken into consideration when standards of measurements procedures and assessments of luminaires and light sources with respect to TML are being constructed.

If temporal variations of a TLA value are observed, it is recommended to declare the highest occurring value.

\section{Acknowledgement}

This work is partly funded by the project "Global Test of SSL Products - IEA-4E-SSL" (J.nr.: 64014-0526) within the Energy Technology Development and Demonstration Program (EUDP), under The Danish Energy Agency.

\section{References}

WILKINS, A., VEITCH, J., \& LEHMAN, B. 2010. LED lighting flicker and potential health concerns: IEEE standard PAR1789 update. Atlanta, GA.

KELLY, D. H. 1964. Sine waves and flicker fusion. Documenta Ophthalmologica vol. 18.

PERZ, M., I. VOGELS, AND D. SEKULOVSKI. 2013. Evaluating the visibility of temporal light artefacts. Lux Europa 2013: 12th European Lighting Conference, Jagiellonian University Krakow, Poland

BULLOUGH, J. D. et al. 2011. Effects of flicker characteristics from solid-state lighting on detection, acceptability and comfort. Lighting Research and Technology, vol. 43, pp. 337348

PERZ, M. et al. 2014. Modelling the visibility of the stroboscopic effect occurring in temporally modulated light systems. Lighting Research and Technology, vol. 0, pp.1-20

ROBERTS, J. E., AND A. J. WILKINS. 2012. Flicker can be perceived during saccades at frequencies in excess of $1 \mathrm{kHz}$. Lighting Research and Technology, vol. 45, pp. 124-132

CIE 2016. CIE TN006:2016. Visual Aspects of Time-Modulated Lighting Systems - Definitions and Measurement Models. 